\title{
ABUNDANCES OF HELIUM IN GASEOUS NEBULAE
}

\author{
M. J.SEATON \\ Department of Physics, University College, London, U.K.
}

During the past two or three years much work has been done on the determination of helium abundances in gaseous nebulae, using observations at both optical wavelengths and radio wavelengths. I imagine that the organisers of this Discussion must have asked whether the subject should be reviewed by an optical observer or a radio observer, and have finally resolved their dilemma by choosing a speaker who has not made observations at any wavelength whatsoever.

\section{Summary of Results}

I will summarise the results obtained before discussing in more detail the various techniques and special problems involved. Let $y$ be the percentage abundance of helium atoms, relative to the number of hydrogen atoms:

$$
y=100 \times \frac{N(\mathrm{He})}{N(\mathrm{H})} .
$$

The helium abundance as a fraction of the total mass, for a mixture of hydrogen and helium, is

$$
Y=0.04 y /(1+0.04 y) \text {. }
$$

A concise summary of results is given in Table I. For $\mathrm{H}^{+}$regions $*$ I adopt $y=9 \pm 2$.

TABLE I

Summary of results

\begin{tabular}{lr} 
Objects & \multicolumn{1}{c}{$y$} \\
\hline $\mathrm{H}^{+}$regions, galactic and extra-galactic & $9 \pm 2$ \\
Planetary Nebulae & $12 \pm 3$
\end{tabular}

Values of $y$ from optical observations tend to be slightly larger than those obtained from radio observations, but the discrepancy is not serious. Observations have been made for galactic $\mathrm{H}^{+}$regions, for a number of galaxies in the local group, and for two galaxies not in the local group. To within probable observational errors, it would appear that $y$ is essentially the same for all these objects. The galaxy NGC 6822 is of interest as a member of the local group with a small mass, a large fraction by mass of interstellar $\mathrm{H}$, and low abundances of $\mathrm{N}$ and $\mathrm{O}$; for this galaxy optical measurements give $y=9.4$ (Peimbert and Spinrad, 1970a). Some evidence has been presented for

* I refer to ' $\mathrm{H}^{+}$regions' rather than 'HII regions', since the Roman numerals should be used only for the designation of spectra. 
variations in $y$ at different points in the galaxy M31 (Schmidt, 1962; Rubin and Ford, 1968): I will leave it to the observers to discuss whether these effects are real.

All determinations of $y$ in planetary nebulae have been made at optical wavelengths. I adopt $y=12 \pm 3$. Is there any significant difierence between the values of $y$ for planetary nebulae and for galactic $\mathrm{H}^{+}$regions? The comparisons should be made using the same observing techniques. The most recent photo-electric observations show that the results for planetaries and $\mathrm{H}^{+}$regions are in quite close agreement.

The planetary observed in the globular cluster M15 (O'Dell et al., 1964) is of interest in providing information about the He abundance in Population II. This planetary has low abundances of $\mathrm{O}$ and $\mathrm{N}$, but a normal value of $y(y=13)$. The planetaries are formed by ejection of material from the outer envelopes of highly evolved stars and this material could be He-rich. However, the close agreement between $\mathrm{He}$ abundances in galactic planetaries and galactic $\mathrm{H}^{+}$regions indicates that any such effect must be small, and it is therefore reasonable to assume that the $\mathrm{He}$ abundance observed in the globular cluster planetary does not differ significantly from the abundance in the material out of which the cluster was formed. The evidence is that the He abundance is the same in Populations I and II. Further support comes from the work of Miller (1969), who obtains $y=13$ for a distant planetary in a direction close to that of the galactic pole, and which also presumably belongs to Population II.

\section{Theory}

Helium abundances in gaseous nebulae are obtained from the interpretation of recombination spectra of hydrogen and helium. An ion $\mathrm{X}^{+}\left(\mathrm{X}^{+}=\mathrm{H}^{+}, \mathrm{He}^{+}\right.$or $\left.\mathrm{He}^{++}\right)$ undergoes radiative recombination,

$$
X^{+}+e \rightarrow X_{n}+h v
$$

to give an atom $\mathrm{X}$ in some excited state $n$, which may then emit further radiation in spectrum lines,

$$
\mathrm{X}_{n} \rightarrow \mathrm{X}_{n^{\prime}}+h v_{n n^{\prime}}
$$

In order to calculate the emissivity in a particular spectrum line one must take account of all relevant radiative and collisional processes; the theory of such calculations is reviewed by Seaton (1968). From the observed intensities of HeI and He II lines, relative to $\mathrm{HI}$ lines, one deduces a quantity

$$
y^{\prime}=100 \frac{\int N_{e}\left\{N\left(\mathrm{He}^{+}\right)+N\left(\mathrm{He}^{++}\right)\right\} \mathrm{d} V}{\int N_{e} N\left(\mathrm{H}^{+}\right) \mathrm{d} V}
$$

where the integrals are over the observed volume. In high excitation planetaries a significant fraction of the $\mathrm{He}$ may be $\mathrm{He}^{++}$. In most other nebulae $\mathrm{He}$ II lines are weak or absent and $\mathrm{He}^{++}$may be neglected. It is more difficult to allow for the fact 
that an appreciable amount of neutral $\mathrm{He}, \mathrm{He}^{0}$, may exist in regions of ionized hydrogen. The ionization potential of $\mathrm{He}^{0}(24.6 \mathrm{eV})$ is much larger than that of $\mathrm{H}^{0}$ $(13.6 \mathrm{eV})$. Consider a star radiating as a black body, at temperature $T_{s}$, completely surrounded by an optically thick nebulae. For small values of $T_{s}$ the number of stellar quanta capable of ionizing $\mathrm{He}^{0}$ is much smaller than the number capable of ionizing $\mathbf{H}^{0}$. In this case one has two ionized regions, an inner region containing $\mathrm{H}^{+}$and $\mathrm{He}^{+}$ and an outer region containing $\mathrm{H}^{+}$and $\mathrm{He}^{0}$. Observations of such nebula will give $y^{\prime}<y$. As $T_{\mathrm{s}}$ increases, the size of the $\left(\mathrm{H}^{+}, \mathrm{He}^{0}\right)$ region decreases, relative to the size of the total $\mathrm{H}^{+}$region. The $\left(\mathrm{H}^{+}, \mathrm{He}^{0}\right)$ region no longer exists for $T_{s}$ larger than some critical value $T_{c}$; for $y=10, T_{c}=4 \times 10^{4} \mathrm{~K}$. The theory, which was first discussed in detail by Hummer and Seaton (1964), is confirmed by observations of planetary nebulae (Harman and Seaton, 1966). Determining $y$ from observations of high excitation planetaries, one obtains $y^{\prime}<y$ for a number of low excitation planetaries, having $T_{s}<T_{c}$.

The stars exciting diffuse $\mathrm{H}^{+}$regions will generally have $T_{s}<T_{c}$. The interpretation of observations is complicated by the fact that the diffuse $\mathrm{H}^{+}$regions have very irregular shapes and, in at least some directions, will not be optically thick. One may measure $\mathrm{He} / \mathrm{HI}$ intensity ratios at various points in a nebula or, even better, obtain iso-photal contours in monochromatic $\mathrm{He}$ and $\mathrm{HI}$ radiations. Observations of Orion give small values of $y^{\prime}$ at points near the edge of the nebula, which shows that there must be some outer regions containing $\mathrm{H}^{+}$and $\mathrm{He}^{0}$ (Peimbert and Costero, 1969). One may attempt to allow for the presence of $\left(\mathrm{H}^{+}, \mathrm{He}^{0}\right)$ regions on constructing three-dimensional models. Use may also be made of the intensities of various forbidden lines. The [SII] lines give a particularly useful guide to the amount of $\mathrm{He}^{0}$, since the ionization potential of $\mathrm{S}^{+}(23.4 \mathrm{eV})$ is a bit smaller than that of $\mathrm{He}^{0}$ (Peimbert and Spinrad, 1970). In using [SII] line intensities to estimate the amount of $\mathrm{He}^{0}$, the main uncertainty may arise from the fact that the [SII] line emissivities are sensitive to electron temperature.

\section{The Interpretation of Optical Observations}

Abundances are usually deduced from the intensities of helium triplet lines, since each triplet line is about three times as intense as the corresponding singlet line. Some complications in the interpretation of the triplet spectrum arise from the metastability of $2{ }^{3} S$. Self-absorption can occur in the lines $2{ }^{3} S \rightarrow n{ }^{3} P$, and $2{ }^{3} P$ can be excited by collisions from $2{ }^{3} S$. Fortunately, these processes do not influence significantly the intensities of the lines $n^{3} D \rightarrow 2{ }^{3} P$. Helium abundances are usually deduced from measured intensities of HeI $3{ }^{3} D \rightarrow 2{ }^{3} P, \lambda 5876$ or $4{ }^{3} D \rightarrow 2{ }^{3} P, \lambda 4471$.

Calculations for $\mathrm{HeI}$ have been made in the following approximations:

(i) Using hydrogenic recombination rates and transition probabilities (Seaton, 1960). For values of the principal quantum number $n>12$ it was assumed that collisions produced a complete redistribution among the levels of different angular momentum quantum number $l$ (Burgess, 1958). 
(ii) As in (i), but neglecting all collisional redistribution in $/$ (Pengelly, 1964).

(iii) Using helium recombination rates and transition probabilities, neglecting collisional redistribution in $l$ (Pengelly, 1963). These results are quoted by Harman and Seaton (1966) and by Seaton (1968).

(iv) As in (iii), but making explicit allowance for collisional transitions between l-states (Robbins, 1968).

The results obtained in approximations (ii) and (iv) are in good agreement, and the calculated intensity ratios $I(5876) / I(4471)$ are in agreement with results of photoelectric observations (Peimbert and Costero, 1969).

A crucial test of the theory is that the calculated relative intensities in each spectrum should be in agreement with the observed relative intensities. A number of discrepancies have been reported between calculated intensities and intensities measured using photographic techniques. Such discrepancies could be due to errors in the theory or errors in the observations. Recent photo-electric work suggests that the discrepancies may be due to errors in photographic photometry such that the intensities of the weaker lines are too large. Further work is much to be desired, since one cannot have complete confidence in any results deduced from optical observations so long as there is doubt as to the measure of agreement between calculated and observed relative intensities in each spectrum.

Harman and Seaton (1966) consider 15 planetaries for which they would expect to have $y^{\prime}=y$, and deduce a mean value of $y=16$. The same date has been reconsidered by Osterbrock (1970), who selects the eight objects for which the most accurate photo-electric observations are available, and obtains $y=13$. Further photo-electric observations have been made by Peimbert and Torres-Peimbert (1970) who obtain $y=11.5 \pm 2$ for 10 planetaries. The discrepancy between photo-electric and photographic results is consistent with the overestimation of photographic intensities of weaker lines.

References to work on $\mathrm{He}$ abundances in $\mathrm{H}^{+}$regions are given in a review by Osterbrock (1970) and I will therefore cite only the most recent papers. From photoelectric observations of three galactic $\mathrm{H}^{+}$regions (Orion, M8 and M17), Peimbert and Costero (1969) obtain $y=11$. The most recent work on $\mathrm{H}^{+}$regions in other galaxies is that of Peimbert and Spinrad (1970a, b).

\section{The Interpretation of Radio Observations}

The lines observed at radio wavelengths are due to transitions, $n \rightarrow n^{\prime}$, between highly excited states. For a given transition the frequency of the Her line differs from that of the Hi line due to the differences in the nuclear masses.

The number of atoms $N_{n}$ in an excited state is expressed in terms of the number for conditions of Saha equilibrium,

$$
N_{n}=b_{n} N_{n} \text { (Saha). }
$$

The factors $b_{n}$ must be calculated allowing for radiative and collisional processes. 
The most accurate calculations are those of Brocklehurst (1970). For given values of electron temperature $T_{e}$ and electron density $N_{e}$ the factors $b_{n}$ will be the same for highly excited states of hydrogen and helium. Calculations show that $b_{n}>b_{n^{\prime}}$ for $n>n^{\prime}$, that is to say the upper levels are overpopulated with respect to the lower levels. It follows that partial maser action can occur (Goldberg, 1966). The intensity in the free-free continuum is large compared with the intensities in the lines, and the maser process therefore involves stimulated emission of line photons by continuum photons; the line intensities depend on $T_{e}, N_{e}$ and continuum optical depth, $\tau_{c}$. As in the optical case, one must consider whether the observed relative intensities in each spectrum are in agreement with theory. It is found that maser action does occur in many nebulae. One can determine values of $T_{e}, N_{e}$ and $\tau_{c}$ such that all of the observed line intensities agree with theory, to within the probable accuracy of the observations, but it is found that the values of $\tau_{c}$ required are larger than the values deduced from observed continuum surface brightnesses (Hjellming and Davies, 1970; Gordon, 1970). A plausible explanation of the discrepancies in $\tau_{c}$ is that much of the emission comes from localised regions of high surface brightness, with angular diameters too small to be resolved at radio wavelengths.

We may now discuss the determination of $\mathrm{He} / \mathrm{H}$ abundance ratios. Three cases may be considered:

(i) If no maser action occurs, the integrated $\mathrm{HI}$ and $\mathrm{HeI}$ line intensities are proportional to $\mathrm{H}^{+}$and $\mathrm{He}^{+}$abundances, and we therefore obtain

$$
\frac{I\left(n \rightarrow n^{\prime}, \mathrm{He}\right)}{I\left(n \rightarrow n^{\prime}, \mathrm{HI}\right)} 100=y^{\prime}
$$

(it is here assumed that $\mathrm{He}^{++}$can be neglected).

(ii) If we have a $\left(\mathrm{H}^{+}, \mathrm{He}^{+}\right)$region but no $\left(\mathrm{H}^{+}, \mathrm{He}^{0}\right)$ region,

$$
\frac{I\left(n \rightarrow n^{\prime}, \mathrm{He} \mathrm{I}\right)}{I\left(n \rightarrow n^{\prime}, \mathrm{HI}\right)} 100=y .
$$

In this case, maser action will enhance the HeI and Hı lines by the same fractional amount and Equation (8) remains valid even if maser action does occur.

(iii) If we have both $\left(\mathrm{H}^{+}, \mathrm{He}^{+}\right)$and $\left(\mathrm{H}^{+}, \mathrm{He}^{0}\right)$ regions in the line of sight, and maser action occurs, then the situation is more complicated. This case does not appear to have been discussed previously. Consider the case that we have a $\left(\mathrm{H}^{+}, \mathrm{He}^{0}\right)$ region in front of a $\left(\mathrm{H}^{+}, \mathrm{He}^{+}\right)$region. Maser action in the $\mathrm{HeI}$ lines then depends on the value of $\tau_{c}$ for the $\left(\mathrm{H}^{+}, \mathrm{He}^{+}\right)$region alone, while maser action in the $\mathrm{HI}$ lines depends on the value of $\tau_{c}$ for both regions combined. In this case we obtain

$$
\frac{I\left(n \rightarrow n^{\prime}, \mathrm{He}\right)}{I\left(n \rightarrow n^{\prime}, \mathrm{HI}\right)} 100<y^{\prime} .
$$

The observational evidence is that the intensity ratios $I\left(n \rightarrow n^{\prime}, \mathrm{HeI}\right) / I\left(n \rightarrow n^{\prime}, \mathrm{HI}\right)$ are the same for lines which show maser action and for lines which do not show maser action (Mezger et al., 1970a and b; Gordon, 1970). This has been taken to provide 
a good check on the theory. It also indicates that there is no very extensive $\left(\mathrm{H}^{+}, \mathrm{He}^{0}\right)$ region contributing to the observed emission. It would be of interest to obtain, from the observational material, an upper limit on the possible contributions from $\left(\mathrm{H}^{+}\right.$, $\mathrm{He}^{0}$ ) regions.

Results of radio determinations of $y^{\prime}$ are summarised in Table II. Careful com-

TABLE II

Values of $y^{\prime}$ obtained from radio observations

\begin{tabular}{lll}
\hline Object & $y^{\prime}$ & Reference \\
\hline Five galactic $\mathrm{H}^{+}$regions & $8.4 \pm 0.3$ & Palmer et al., 1969 \\
Northern galactic $\mathrm{H}^{+}$regions & 8 & Reifenstein et al., 1970 \\
Southern galactic $\mathrm{H}^{+}$regions & 8.8 & Mezger et al., 1970a \\
Orion Nebula (M42) & $7.3 \pm 0.8$ & Gordon, 1970 \\
Orion Nebula (M42) & $8.1 \pm 0.2$ & \\
Lagoon Nebula (M8) & $9.3 \pm 2.9$ & Churchwell and Mezger, 1970 \\
Omega Nebula (M17) & $9.4 \pm 0.6$ & \\
30 Doradus nebula (LMC) & $17 \pm 9$ & Mezger et al., 1970b
\end{tabular}

parisons of optical and radio determinations of $y^{\prime}$ have been made (Churchwell and Mezger, 1970), particularly for the Orion nebula. Agreement is generally good, but there may be some tendency for the optical values of $y^{\prime}$ to be slightly larger than the radio values. It should also be noted that estimated corrections for $\mathrm{He}^{0}$ in the ionized regions have been made in several recent papers by optical observers, but not in the work of the radio observers.

\section{Conclusions}

The accuracy of the best optical determinations of $\mathrm{He} / \mathrm{H}$ abundance ratios is probably comparable to that of the best radio determinations. One advantage of optical work is that observations can be made on sources with small total flux (planetary nebulae and extra-galactic objects).

Further careful comparisons of radio and optical results should be made for selected objects. In order to gain complete confidence in the interpretation of the observations it is also highly desirable to make further careful studies of the relative intensities in each spectrum, at both radio and optical wavelengths.

So far there is no very convincing evidence for variations in $y$ between different objects. Once again, further observational work is required.

\section{References}

Brocklehurst, M.: 1970, Monthly Notices Roy. Astron. Soc. 148, 417.

Burgess, A.: 1958, Monthly Notices Roy. Astron. Soc. 118, 477.

Churchwell, E. and Mezger, P. G.: 1970, Astrophys. Letters 5, 227.

Goldberg, L.: 1966, Astrophys. J. 144, 1225.

Gordon, M. A.: 1970, Astrophys. Letters 6, 27. 
Harman, R. J. and Seaton, M. J.: 1966, Monthly Notices Roy. Astron. Soc. 132, 15.

Hjellming, R. M. and Davies, R. D.: 1970, Astron. Astrophys. 5, 53.

Hummer, D. G. and Seaton, M. J.: 1964, Monthly Notices Roy. Astron. Soc. 127, 217.

Mezger, P. G., Wilson, T. L., Gardner, F. F., and Milne, D. K.: 1970a, Astrophys. Letters 6, 35.

Mezger, P. G., Wilson, T. L., Gardner, F. F., and Milne, D. K.: 1970b, preprint.

Miller, J. S.: 1969, Astrophys. J. 157, 1215.

O'Dell, C. R., Peimbert, M., and Kinman, T. D.: 1964, Astrophys. J. 140, 119.

Osterbrock, D. E.: 1970, Quart. J. Roy. Astron. Soc. 11, 199.

Palmer, P., Zuckerman, B., Penfield, H., and Lilley, A. E.: 1970, Astrophys. J. 156, 887.

Peimbert, M. and Costero, R.: 1969, Bol. Obs. Tonantzintla y Tacubaya 5, 3.

Peimbert, M. and Spinrad, H.: 1970a, Astron. Astrophys. 7, 311.

Peimbert, M. and Spinrad, H.: 1970b. Astrophys. $J$.

Peimbert, M. and Torres-Peimbert, S.: 1970, to be submitted to Astrophys. $J$.

Pengelly, R. M.: 1963, Thesis, Univ. London.

Pengelly, R. M.: 1964, Monthly Notices Roy. Astron Soc. 127, 145.

Reifenstein, E. C., Wilson, T. L., Burke, B. F., Mezger, P. G., and Altenhoff, W. J.: 1970, Astron. Astrophys. 4, 357.

Robbins, R. R.: 1968, Astrophys. J. 151, 497.

Rubin, V. and Ford, W. K.: 1968, Interstellar Ionized Hydrogen (ed. by Y. Terzian), Benjamin, New York, p. 641.

Schmidt, M.: 1962, Symposium on Stellar Evolution, La Plata Observatory, Argentina, p. 61.

Seaton, M. J.: 1960, Rept. Progr. Phys. 23, 313.

Seaton, M. J.: 1968, Adv. Atomic Molec. Phys. 4, 331.

\section{DISCUSSION}

D. E. Osterbrock: Dr Vera Rubin is unable to attend our session, but would like me to read the following note:

"Spectra of $50 \mathrm{HII}$ regions in M31 have been observed, with distances from the nucleus ranging from 3 to $24 \mathrm{kpc}$. The line $\mathrm{He} \lambda 5876$ is in the spectral region observed.

(1) For 15 regions, the observed line ratio $\lambda 5876 / \mathrm{H} \alpha$ ranges from 0.01 to 0.08 (with an uncertainty of about $30 \%$ for each value). This implies $N(\mathrm{He}) / N(\mathrm{H})$ ranges from $2 \%$ to $15 \%$ by number. The mean value for 15 regions is $12 \%$. There is a large scatter at each $R$, but no systematic variation with distance from the nucleus.

(2) For 21 regions $N(\mathrm{He}) / N(\mathrm{H})$ is less than $3 \%$ (i.e. no line observed on well exposed plate). These 21 regions are at all distances from nucleus.

(3) For these $15+21$ regions, mean $N(\mathrm{He}) / N(\mathrm{H})<0.08$. Note that for $[\mathrm{N} I 1] / \mathrm{H}$ and $[\mathrm{S} \mathrm{II}] / \mathrm{H}$ line ratios, there is a strong dependence on $R$, i.e. both line ratios are largest for regions near the nucleus, and decrease with increasing $R . "$

$M$. Schwarzschild: Dr Seaton's reference to the planetary nebula in M15 seems to me to be of special interest. In the commission for stellar structure, Dr Paczinski has given us a most fascinating report on his computations regarding the ejection of a planetary nebula from a red giant, caused by releasing the ionization energy of $\mathrm{H}$ and $\mathrm{He}$ within the extended envelope. His results make it appear plausible that purely unmixed and unburned matter is ejected by this mechanism. If this is true, then the observed 'normal' He abundance of the planetary nebula in M15 should give us directly the initial He abundance of this cluster. On this basis I think $\mathbf{I}$ am now ready to bet that our Galaxy started with a substantial, i.e. near 'normal', abundance of He.

L. H. Aller: There is an acute need for additional improved observational data. Czyzak and I have tried to calibrate all our photographic line intensity measurements by photoelectric photometry; in particular the $\mathrm{I}(4471) / \mathrm{I}(\mathrm{H} \beta)$ ratio is measured photoelectrically. Of course, the photoelectric calibration should be extended to the fainter lines.

Effects of filamentary structure are particularly troublesome. One needs for high resolution direct photographs secured with narrow bandpass filters in the radiation of [SII], [OH], $\lambda 4471$ and $\mathrm{H} \beta$. Huge fluctuations in $N_{e}$ and $T_{e}$ can occur and will affect the $\mathrm{H}$ and Her emissions differently. Note that photoelectric observations integrate over large surface areas and thus possibly tend to smooth over fluctuations. 
If collisional excitation can occur, note that the effects will be markedly different for $\mathrm{H}$ and $\mathrm{He}$ because of the differences in term structure.

Finally, mention may be made of the work of Kaler who employed all available observations of line intensities and found $\mathrm{He} / \mathrm{H}$ ratios in the range $0.09<\mathrm{He} / \mathrm{H}<0.20$. This ratio appeared to be inversely correlated with the $\mathrm{O} / \mathrm{H}$ ratio.

Roger Cayrel: I want to support the view expressed by Dr. Schwarzschild that it seems somewhat unlikely that the high helium of the planetary nebule in M15 found by O'Dell, Peimbert, and Kinman is produced within the nebula.

It would be a strange coincidence that the central star had managed to put into its expelled shell exactly the proper amount of $\mathrm{He}$ found in all other planetaries from an initially helium-poor matter. One would expect more likely to have much more or much less. 